\title{
A Comprehensive Study on the Dynamic Range of Wavelength Exchange and Its Impact on Exchanged Signal Performance
}

\author{
Mengzhe Shen, Henry King Yin Cheung, Rebecca Wai Lam Fung, and Kenneth Kin-Yip Wong, Member, IEEE
}

\begin{abstract}
Effects of the incomplete signal exchange on the dynamic range performance of type II wavelength exchange (WE II, i.e. both pumps are in anomalous dispersion region) are investigated analytically and experimentally. The dynamic range here is defined as the tolerable operational range of the signal power to maintain a nearly complete WE performance. We studied the case of equal signal powers (balanced signal powers) as well as the case of unequal signal powers (imbalanced signal powers). It is demonstrated that the beating between exchanged signal and residual one and the extinction ratio degradation due to the existence of residual power at original channel is the major source of the signal quality degradation after WE II. The experimental results demonstrated dynamic range $\sim 17 \mathrm{~dB}$ with bit-error rate $(\mathrm{BER})<10^{-9}$ could be maintained with power penalty of $<2 \mathrm{~dB}$ in the case of balanced signal powers. In the case of imbalanced signal powers, the dynamic range was $>8 \mathrm{~dB}$ with the power penalty incurred of $\sim 2 \mathrm{~dB}$ in the worst case.
\end{abstract}

Index Terms-Four-wave mixing (FWM), parametric wavelength exchange (PWE).

\section{INTRODUCTION}

W AVELENGTH exchange (WE) relies on four-wave mixing (FWM) phenomenon in highly nonlinear dispersion-shifted fibers (HNL-DSF) and it has been extensively studied in recent research [1]-[4]. Simultaneous swapping of two signals could be achieved by a suitable choice of wavelengths of both continuous-wave (CW) pumps either at the normal dispersion region (denoted as Type I WE or WE I) [2] or the anomalous dispersion region (denoted as Type II WE or WE II) [4]. Past results showed that the pump-induced Raman amplification introduced asymmetric power transfer that degraded the performance of the WE I process. Such performance degradation was particularly severe when the two pumps were arranged orthogonally in the normal dispersion region [2]. Thus, WE II configuration with two pumps in the anomalous dispersion regime was presented [4]. In theory, no Raman gain is provided by pumps allocated in anomalous dispersion region.

Manuscript received September 19, 2008; revised December 18, 2008 and January 15, 2009. First published April 24, 2009; current version published July 15, 2009. This work was support in part by the Grants Council of the Hong Kong Special Administrative Region, China, under Projects HKU 7172/07E and HKU 7179/08E.

The authors are with the Department of Electrical and Electronic Engineering, the University of Hong Kong, Hong Kong, China (e-mail: mzshen@eee.hku.hk; kycheung@eee.hku.hk; wlfung@eee.hku.hk; kywong@eee.hku.hk).

Color versions of one or more of the figures in this paper are available online at http://ieeexplore.ieee.org.

Digital Object Identifier 10.1109/JLT.2009.2014974
Therefore, it is expected that with this arrangement, signals in the normal dispersion region will exhibit symmetric power transfer characteristics and a nearly complete wavelength exchange can be achieved. We have recently investigated the tuning range of WE II in the anomalous-dispersion region [4], where we focussed on the tuning range by fixing one pump wavelength and its corresponding signal wavelength while tuning the other pump and its corresponding signal wavelength. A tuning range of $15 \mathrm{~nm}$ can be achieved with performance slightly degraded when one of the signals was tuned near the zero-dispersion wavelength (ZDW) of the fiber. WE II may find many applications in modern optical communication networks, such as all-optical packet switching [5], [6] and optical time-division multiplexing (OTDM) [7]-[9]. Thus, it is necessary to have a comprehensive study on its performance. For the practical use of WE II in the optical networks, considerations must be taken into account about the extent of signal quality performance after WE II. In [4], a bit-error rate (BER) of $<10^{-9}$ was recorded with power penalties of $\sim 2 \mathrm{~dB}$. Previous work [2] demonstrated that the main source of the noise in the exchanged signal is the coherent crosstalk between the exchanged signal and the residual power at the same wavelength. Another factor that affects the exchanged signals' qualities of WE II is its relatively low conversion ratio (CR), which also attributes to the existence of residual power. The highest value recorded so far is $28 \mathrm{~dB}$ [2]. It is evaluated by measuring the power ratio between the idler (exchanged signal at the new wavelength) and the residual signal (at the original wavelength) when there is only one signal input, as shown in Fig. 1(b) [2]. Thus, some obvious questions needed to be addressed include the existence of the residual power at the original wavelength after WE II and whether it is possible to realize complete exchange, i.e., no residual power left at the original wavelength. The next logical question is the mechanism through which the residual signal interferes with the exchanged one.

Furthermore, in practical networking systems, signals arrive at the processing nodes via different routes and thus experience different condition in the transmission link, such as fiber loss, nonlinear effect, and unequal amplification of different regenerators. Therefore, the signals may possess different power levels upon the arrival at the processing node. Thus, it is worthwhile to investigate the operational range between the signal powers to maintain a nearly complete WE performance. We define this operational range as the dynamic range, as shown in Fig. 5.

In this paper, we study for the first time, to the best of our knowledge, the dynamic range of WE II with the two pumps 

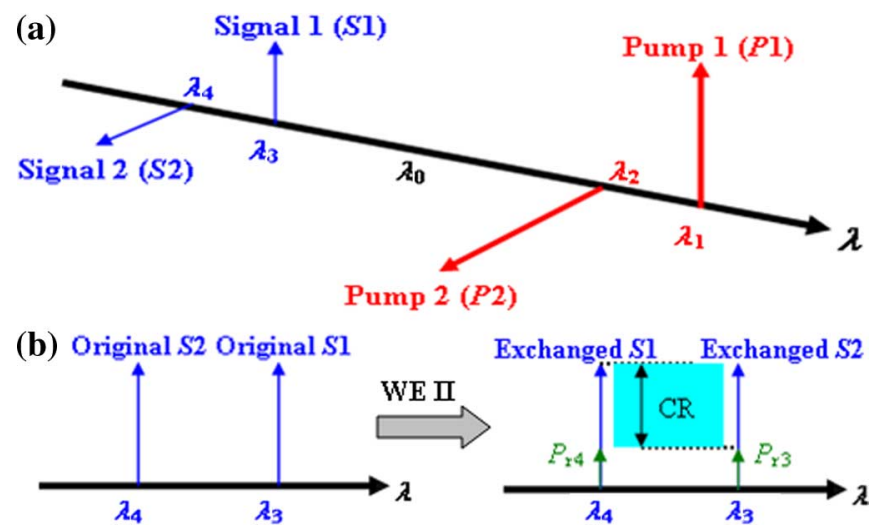

Fig. 1. (a) Wavelength allocation and state-of-polarization of pumps and signals in WE II. (b) Wavelength notation before and after WE II.

( $P 1$ and $P 2$ ) located at the anomalous-dispersion region and the two signals ( $S 1$ and $S 2$ ) located at the normal-dispersion region. We investigate the residual power in WE II and its effects on the exchanged signal quality performance after WE II. We also show that the amount of residual power depend on the phase mismatch condition of WE II for both signals. In Section II, we present the relevant theory and investigate the dynamic range of WE II analytically. We then perform experiments to verify our theoretical prediction followed by discussions in Section III. Finally, concluding remarks are presented in Section IV.

\section{THEORY}

\section{A. Origin of Residual Power}

The form of the basic differential equations describing the WE II was shown in [2] by considering self-phase modulation (SPM), cross-phase modulation (XPM) and four-wave mixing (FWM) interactions among four waves. Here, we consider the case of most interest to us, i.e., when the polarization of neighboring waves are orthogonal with respect to each other (denoted as $\mathrm{WE} \mathrm{II}_{\perp}$ ), as shown in Fig. 1(a). The purpose of orthogonal polarization is to suppress the spurious FWM components. Consider four waves of radian frequencies $\omega_{i}, i=1-4$, and the frequencies satisfy $\omega_{1}+\omega_{4}=\omega_{2}+\omega_{3}$. Waves $1(W 1)$ and 2 (W2) correspond to pumps one (P1) and two (P2), 3 (W3) and $4(W 4)$ are signal one $(S 1)$ and signal two $(S 2)$. One thing noteworthy is that $S 1$ and $S 2$ will exchange the wavelengths of their carriers, i.e., the exchanged $S 1$ and $S 2$ will be at Wave 4 and 3, respectively, after WE II, as shown in Fig. 1(b). Thus, in the following, we will distinguish original $S 1(S 2)$ from exchanged $S 1(S 2)$ for clarity. Assuming that there is no fiber loss and negligible polarization-mode dispersion (PMD) along the fiber, the coupled equations for this configuration are given by [10]

$$
\begin{aligned}
\frac{\partial A_{1}}{\partial z}=i \gamma & {\left[A_{1}^{2}+2 A_{3}^{2}+\frac{2}{3}\left(A_{2}^{2}+A_{4}^{2}\right)\right] A_{1} } \\
+ & \frac{2}{3} i \gamma A_{2} A_{3} A_{4}^{*} \exp (-i \Delta \beta z) \\
\frac{\partial A_{2}}{\partial z}=i \gamma & {\left[A_{2}^{2}+2 A_{4}^{2}+\frac{2}{3}\left(A_{1}^{2}+A_{3}^{2}\right)\right] A_{2} } \\
+ & \frac{2}{3} i \gamma A_{1} A_{3}^{*} A_{4} \exp (i \Delta \beta z)
\end{aligned}
$$

$$
\begin{aligned}
\frac{\partial A_{3}}{\partial z}=i \gamma & {\left[A_{3}^{2}+2 A_{1}^{2}+\frac{2}{3}\left(A_{2}^{2}+A_{4}^{2}\right)\right] A_{3} } \\
+ & \frac{2}{3} i \gamma A_{1} A_{2}^{*} A_{4} \exp (i \Delta \beta z) \\
\frac{\partial A_{4}}{\partial z}=i \gamma & {\left[A_{4}^{2}+2 A_{2}^{2}+\frac{2}{3}\left(A_{1}^{2}+A_{3}^{2}\right)\right] A_{4} } \\
+ & \frac{2}{3} i \gamma A_{1}^{*} A_{2} A_{3} \exp (-i \Delta \beta z)
\end{aligned}
$$

where $A_{j}$ is the slowly varying envelope of the optical field with frequency $\omega_{j}$ and $\gamma$ is the fiber nonlinear coefficient. We define the propagation constant mismatch as $\Delta \beta=\beta_{1}+\beta_{4}-$ $\beta_{2}-\beta_{3}$, where $\beta_{j}$ are the propagation constants in the fiber. The linear phase mismatch $\Delta \beta$ due to chromatic dispersion can be expressed as [1]

$$
\Delta \beta=2 \sum_{m=1}^{\infty} \frac{\beta_{2 m}}{2 m !}\left[\left(\Delta \omega_{4}\right)^{2 m}-\left(\Delta \omega_{3}\right)^{2 m}\right]
$$

In which $\Delta \omega_{3}=\omega_{3}-\omega_{0}, \Delta \omega_{4}=\omega_{4}-\omega_{0}, \beta_{2 \mathrm{~m}}$ denotes the $2 m$ th derivative of $\beta$ with respect to frequency around the ZDW. To gain better insight into $\Delta \beta$ we limit the summation of (2) to $m=1,2$. The $\mathrm{FWM}$ efficiency for $\mathrm{WE} \mathrm{II}_{\perp}$ scheme is $1 / 9$ compared with that when all waves are aligned in SOP [2].

By introducing new variables $A_{j}=B_{j} \exp \left(\phi_{j}\right)$, where $B_{j}$ and $\phi_{j}$ are the amplitude and the phase of the optical field $A_{j}$, (1) can be rewritten as

$$
\begin{aligned}
\frac{\partial B_{j}}{\partial z}= & \frac{2}{3} m_{j} \gamma \frac{B_{1} B_{2} B_{3} B_{4}}{B_{j}} \sin \phi \\
\frac{\partial \phi}{\partial z}= & \Delta \beta-\gamma \sum_{j=1}^{4} m_{j} B_{j}^{2} \\
& +\frac{2}{3} \gamma B_{1} B_{2} B_{3} B_{4} \sum_{j=1}^{4} m_{j} B_{j}^{-2} \cos \phi
\end{aligned}
$$

where $m_{j}=+1(j=1,4),-1(j=2,3)$ and $\phi=\Delta \beta z+$ $\phi_{1}+\phi_{4}-\phi_{2}-\phi_{3}$. As can be observed from (3), while the $\phi$ oscillates between $-\pi / 2$ and $\pi / 2$ along the fiber, the power flows from $W 4$ to $W 3, W 1$ to $W 2$ and vice versa. Assuming the relative phase $\phi=-\pi / 2$, the third term in (4) can be neglected and the following approximation is valid

$$
\frac{\partial \phi}{\partial z}=\Delta \beta-\gamma\left(B_{1}^{2}-B_{2}^{2}-B_{3}^{2}+B_{4}^{2}\right) .
$$

Considering the case that $W 1$ and $W 4$ are losing power and assuming a positive linear phase mismatch $\Delta \beta$, when they are decreasing, the right-hand side term in (5) gets larger. The flow of power will not turn its direction until $\phi$ approaches zero. Ideally, $P_{4}(L)$ oscillates between 0 and $P_{3}(0)+P_{4}(0)$ without considering fiber loss. However, if the power flow reverses its direction before $B_{4}$ approaches 0 , the amount of power existing at $W 4$ when $\phi=0$ will not be transferred to the $W 3$ and constitutes for the residual power at $W 4$. The origin of residual power $P_{r j}(j=3,4)$ is shown in Fig. 2 such that $P_{r 3}=\min \left[P_{3}(L)\right]$ is the residue at $W 3$ and $P_{r 4}=\min \left[P_{4}(L)\right]$ is at $W 4$. In Fig. 2, we calculated the signal output powers $P_{4}(L)$ and $P_{3}(L)$ as a function of fiber length by (3) and (4), in which $\cos \phi_{0}=0$, $\phi_{0}=\phi_{1}+\phi_{4}-\phi_{2}-\phi_{3}$ is the initial phase difference at $z=0$. 


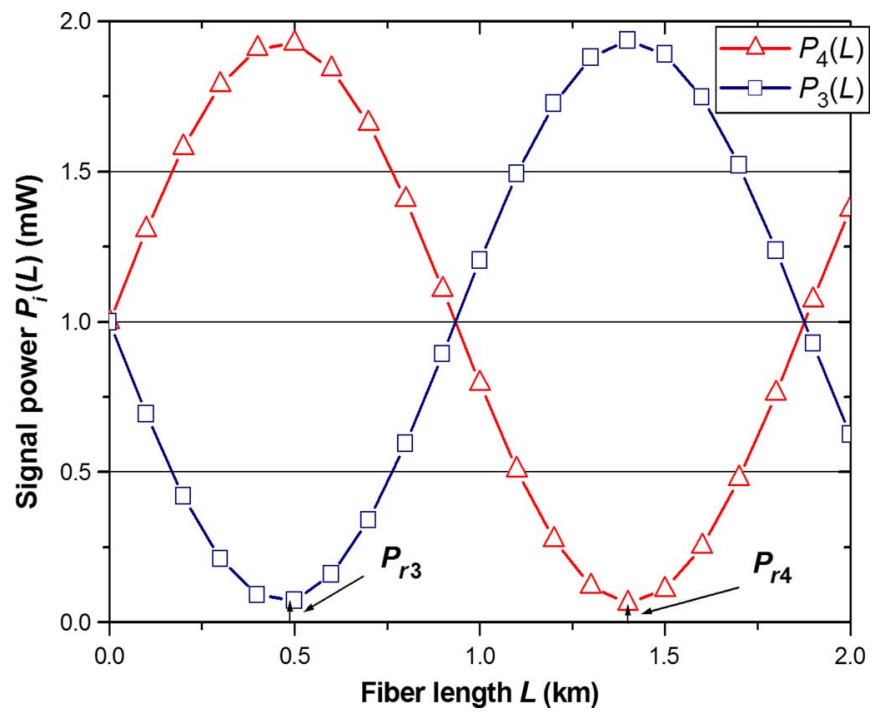

Fig. 2. Signal power variation along the fiber length.

As the initial phase difference $\phi_{0}$ was not controlled in our experimental setup, it is reasonable to set $\cos \phi_{0}$ at its mean value. The fiber parameters used were ZDW $=1541 \mathrm{~nm}, \mathrm{~d} D / \mathrm{d} \lambda=$ $0.03 \mathrm{ps} / \mathrm{nm}^{2} \mathrm{~km}, \beta_{4}=10^{-4} \mathrm{ps}^{4} / \mathrm{km}, \gamma=12 \mathrm{~W}^{-1} \mathrm{~km}^{-1}$. The two signals were located at 1529 (original $S 2$ ) and $1534 \mathrm{~nm}$ (original $S 1$ ) with the initial powers of $0 \mathrm{dBm}$. The two pump powers were located at $1549 \mathrm{~nm}(P 2)$ and $1554 \mathrm{~nm}(P 1)$ with the powers of $20.8 \mathrm{dBm}$, respectively.

\section{B. Q-Factor Penalty}

After defining the residual power and its origin, now we explore how it affects the exchanged signal quality. In the following analysis, we apply the Q-factor as a gauge for the signal quality. If we neglect the thermal noise, the input signal Q-factor for $W_{j}(j=3,4)$ after detection in a receiver is written as [11]

$$
Q_{\mathrm{in}, \mathrm{dB}}=10 l g\left(\frac{I_{1}-I_{0}}{\sqrt{\sigma_{1, S}^{2}+\sigma_{1, \mathrm{ASE}}^{2}}+\sqrt{\sigma_{0, S}^{2}+\sigma_{0, \mathrm{ASE}}^{2}}}\right)
$$

in which the noise contributing factors can be expressed as

$$
\begin{aligned}
\sigma_{1, S}^{2} & =2 q R P_{j}(0) \Delta f \\
\sigma_{0, S}^{2} & =2 q R P_{j}(0) r_{\mathrm{ex}, \mathrm{in}} \Delta f \\
\sigma_{1, \mathrm{ASE}}^{2} & =4 R^{2} G(G-1) P_{j}(0) h \nu \Delta f \\
\sigma_{0, \mathrm{ASE}}^{2} & =4 R^{2} G(G-1) P_{j}(0) r_{\mathrm{ex}, \mathrm{in}} h \nu \Delta f
\end{aligned}
$$

and the signal is

$$
I_{1}-I_{0}=R P_{j}(0)\left(1-r_{\mathrm{ex}, \mathrm{in}}\right)
$$

where $R=q / h \nu$ is the responsivity of the detector with the quantum efficiency $\eta=1$ and $\Delta f$ is the electrical filter bandwidth. $P_{j}(0)$ is the average power of input signal $(j=3$ or 4$)$. $G$ is the gain of erbium-doped fiber amplifier (EDFA) with the spontaneous-emission factor $n_{s p}=1 . r_{\mathrm{ex}, \text { in }}=P_{\mathrm{off}} / P_{\mathrm{on}}$ is the extinction ratio of input signal, where $P_{\text {off }}$ is the power carried by "0" bits and $P_{\text {on }}$ is the power carried by " 1 " bits. Defining the extinction ratio in which the smaller the better is to avoid infinity in derivation [11]. The root mean square (RMS) noise currents $\sigma_{1}$ and $\sigma_{0}$ include the contributions of both shot noise and the amplified spontaneous emission (ASE) noise from EDFA.

Sources of the input signals' Q-factor degradation include the lowered extinction ratio due to the existence of residual power after WE II. They will be the most detrimental if the exchange happens between " 0 " bits and "1" bits in the two wavelengths because the extinction ratio falls significantly at that time instant. On the contrary, impact of the exchange between "1" and "1" manifests itself as the beating of residual power with exchanged signal. The residual power mixes with the exchanged signal and produces the current $I=R\left(E_{r 7-j}+E_{7-j}(L)\right)^{2}$ ( $j=3$ or 4 ) at the photodetector with responsivity $R$. Noting that $E_{r 7-j}$ and $E_{7-j}(L)$ oscillate at same frequency with a random phase difference. The intensity variations after detection would be

$$
\sigma_{1, e x-r e} \approx \sqrt{R^{2} P_{7-j}(L) P_{r 7-j}}
$$

The output Q-factor can now be obtained as

$$
\begin{aligned}
& Q_{\text {out }, \mathrm{dB}} \\
& =10 \lg \left(\frac{I_{1}-I_{0}}{\sqrt{\left(\sigma_{1, s}^{2}+\sigma_{1, e x-r e}^{2}+\sigma_{1, \mathrm{ASE}}^{2}\right)}+\sqrt{\left(\sigma_{0, s}^{2}+\sigma_{0, \mathrm{ASE}}^{2}\right)}}\right)
\end{aligned}
$$

and the signal is

$$
I_{1}-I_{0}=R P_{7-j}(L)\left(1-r_{\text {ex }, \text { out }}\right) .
$$

Combining the input and output Q-factors results the penalty

$$
Q_{\text {penalty }, \mathrm{dB}}=Q_{\mathrm{in}, \mathrm{dB}}-Q_{\text {out }, \mathrm{dB}} .
$$

\section{Phase-Mismatch Condition}

The impact of the imperfect phase matching on the power conversion efficiency was discussed in [1]. In addition, how pump depletion affects the power conversion efficiency was investigated in [2], in which the dependency of maximum extinction ratio on signal power is attained. In this subsection, the phase-mismatch condition for WE II under the assumption of pump depletion is investigated. In the context of WE II, the phase-mismatch condition guarantees that there is no residual power at the original wavelength after WE II, i.e., $P_{r 4}=0$ or $P_{r 3}=0$. According to the analysis about the noise mechanism of WE II mentioned in Section II-B, the residual power affects the exchanged signal performance. To explore the phase-mismatch condition of WE II, we adopt the approach in [12], [13] to attain the exact solution for (1). Our starting point is (3) and (4). With variable transformations

$$
\Gamma=\frac{2}{3} \gamma P_{0} z, \quad \xi_{j}=\frac{B_{j}}{\sqrt{P_{0}}}
$$


where $P_{0}=B_{1}^{2}(0)+B_{2}^{2}(0)+B_{3}^{2}(0)+B_{4}^{2}(0)$ representing the total power. Eq. (16) converts (3) and (4) into normalized coupled equations

$$
\begin{aligned}
\frac{\partial \xi_{j}}{\partial \Gamma} & =m_{j} \frac{\xi_{1} \xi_{2} \xi_{3} \xi_{4} \sin \phi}{\xi_{j}} \\
\frac{\partial \phi}{\partial \Gamma} & =\frac{3 \Delta \beta}{2 \gamma P_{0}}-\frac{3}{2} \sum_{j=1}^{4} m_{j} \xi_{j}^{2}+\xi_{1} \xi_{2} \xi_{3} \xi_{4} \sum_{j=1}^{4} m_{j} \xi_{j}^{-2} \cos \phi .
\end{aligned}
$$

From (17) and (18) we can obtain four more invariants besides the conservation of total power

$$
\begin{aligned}
x_{1} & =\xi_{1}^{2}-\xi_{4}^{2} \\
x_{2} & =\xi_{2}^{2}+\xi_{4}^{2} \\
x_{3} & =\xi_{3}^{2}+\xi_{4}^{2} \\
Z & =\frac{3 \Delta \beta}{4 \gamma P_{0}} \xi_{4}^{2}+\xi_{1} \xi_{2} \xi_{3} \xi_{4} \cos \phi-\frac{3}{8}\left(\xi_{1}^{4}+\xi_{2}^{4}+\xi_{3}^{4}+\xi_{4}^{4}\right) .
\end{aligned}
$$

The solution of (3) and (4) can be acquired by the integration of

$$
\frac{\partial \xi_{4}}{\partial \Gamma}=\xi_{1} \xi_{2} \xi_{3} \sin \phi
$$

After substitution of (19) into (20), the resultant integral is

$$
\Gamma= \pm \frac{1}{2} \int_{x(0)}^{x(\Gamma)} \frac{d x}{\sqrt{f(x)}} .
$$

The quartic polynomial function $f(x)$ is given by

$$
\begin{aligned}
f(x) & =x\left(x_{1}+x\right)\left(x_{2}-x\right)\left(x_{3}-x\right)-\left\{Z-\frac{3 \Delta \beta x}{4 \gamma P_{0}}\right. \\
+ & \left.\frac{3}{8}\left[\left(x_{1}+x\right)^{2}+\left(x_{2}-x\right)^{2}+\left(x_{3}-x\right)^{2}+x^{2}\right]\right\}^{2}
\end{aligned}
$$

where $\xi_{4}^{2}=P_{4} / P_{0}=x$ are normalized power of original $S 2$. The polynomial function $f(x)=0$ has four roots, which can be ordered as $\eta_{4}>\eta_{3}>\eta_{2}>\eta_{1}$.

It is shown in Fig. 2 that as the waves $W 3$ and $W 4$ propagate down the fiber the powers transfer from $W 3$ to $W 4$ and then back in an oscillating form with a fixed period. As shown by the mathematical derivation in [12], it was claimed that the solution for $x$, which is the normalized $P_{4}(L)$ in (21), will oscillate between the two lowest positive roots $\left(\eta_{1}, \eta_{2}\right)$ of the polynomial equation $f(x)=0$, i.e., $P_{4}(L)$ satisfies $\eta_{1} P_{0} \leq P_{4}(L) \leq \eta_{2} P_{0}$. Inasmuch as the signal power $P_{3}(L)+P_{4}(L)$ is conserved in (19), $\left(x_{3}-\eta_{2}\right) P_{0} \leq P_{3}(L) \leq\left(x_{3}-\eta_{1}\right) P_{0}$, based on the definition of $P_{r 3}$ in Section II-A, $P_{r 3}=\min \left[P_{3}(L)\right]$ and $P_{r 4}=$ $\min \left[P_{4}(L)\right]$. Consequently, $\eta_{1}=0$ is sufficient for achieving $P_{r 4}=0$ and $\eta_{2}=x_{3}$ is sufficient for achieving $P_{r 3}=0$. Thus, the phase-mismatch condition for original $S 1(S 2)$ necessarily requires that $\eta_{2}=x_{3}\left(\eta_{1}=0\right)$ to achieve complete WE II. After some algebra, these relations lead to

$$
\begin{aligned}
\xi_{30}^{2}[ & -\frac{3 \Delta \beta}{4 \gamma P_{0}}+\frac{\xi_{10} \xi_{20} \xi_{40}}{\xi_{30}} \cos \phi_{0} \\
& \left.+\frac{3}{4}\left(\xi_{10}^{2}-\xi_{20}^{2}+\xi_{30}^{2}+\xi_{40}^{2}\right)\right]=0
\end{aligned}
$$

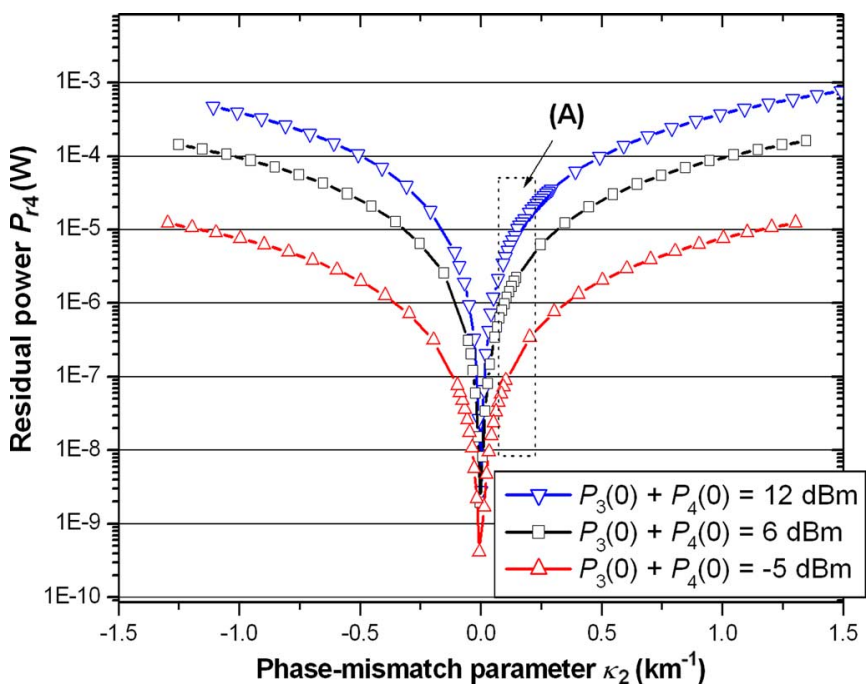

Fig. 3. Residual power $P_{r 4}$ versus the phase-mismatch parameter $\kappa_{2}$.

$$
\begin{aligned}
\xi_{40}^{2}[ & \frac{3 \Delta \beta}{4 \gamma P_{0}}+\frac{\xi_{10} \xi_{20} \xi_{30}}{\xi_{40}} \cos \phi_{0} \\
& \left.+\frac{3}{4}\left(-\xi_{10}^{2}+\xi_{20}^{2}+\xi_{30}^{2}+\xi_{40}^{2}\right)\right]=0
\end{aligned}
$$

where $\xi_{i 0}^{2}$ denotes the respective initial normalized power of signals and pumps. Assuming there are two signals at the input and $\cos \phi_{0}=0,(23)$ and (24) can be simplified

$$
\begin{aligned}
& -\Delta \beta+\gamma\left[P_{1}(0)-P_{2}(0)+P_{3}(0)+P_{4}(0)\right]=0 \\
& \Delta \beta+\gamma\left[-P_{1}(0)+P_{2}(0)+P_{3}(0)+P_{4}(0)\right]=0
\end{aligned}
$$

Equations (25) and (26) provide us the phase-mismatch condition for original $S 1$ and $S 2$. If they are satisfied, there will be no residue from original $S 1$ or $S 2$. Generally, the more power left at the original wavelength, the worse the quality of the exchanged signal. Attentive readers may have noticed that if we add (25) and (26) together, an impractical equation $\gamma\left(P_{3}(0)+P_{4}(0)\right)=0$ is raised. It demonstrates that $S 1$ and $S 2$ cannot be completely exchanged in theory, but we can obtain a much better understanding on the dynamic range of WE through the condition we attain. The mathematical forms of (25) and (26) are very much alike the phase-mismatch condition introduced in fiber optical parametric amplifier (FOPA) [14] because WE II and FOPA are based on similar underlying principle. We can define the left-hand side of (25) and (26) as the phase-mismatch parameter for original $S 1$ and $S 2$, respectively, as

$$
\begin{aligned}
& \kappa_{1}=-\Delta \beta+\gamma\left[P_{1}(0)-P_{2}(0)+P_{3}(0)+P_{4}(0)\right] \\
& \kappa_{2}=\Delta \beta+\gamma\left[-P_{1}(0)+P_{2}(0)+P_{3}(0)+P_{4}(0)\right] .
\end{aligned}
$$

Numerical calculations reveal that the larger $\kappa_{1}$ and $\kappa_{2}$ deviate from zero, the larger the residual power at the corresponding wavelength, as is shown in Fig. 3. In Fig. 3, pump powers and fiber parameters were the same as those used in Fig. 2 except that the fiber length was $1 \mathrm{~km}$. It shows the residual power $P_{r 4}$ as a function of phase-mismatch parameter $\kappa_{2}$. Note that it is an even function of $\kappa_{2}$. It also compares the residual power $P_{r 4}$ among various signal input power. For signals having the same $\kappa_{2}$, higher initial power will result in higher residue. The dotted 


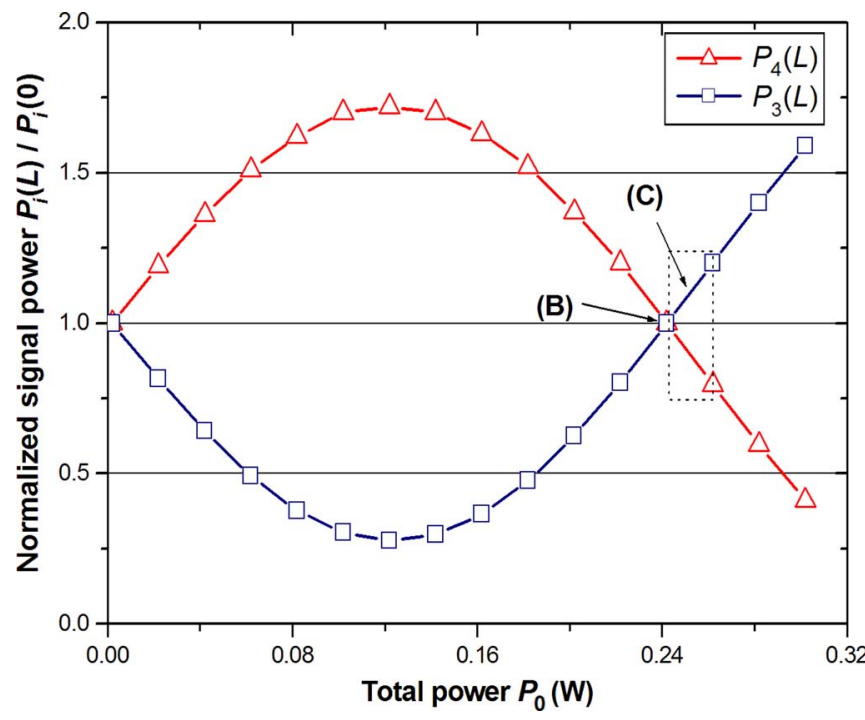

Fig. 4. Normalized signal output power transfer characteristics.

box "A" plots the approximate magnitude of residual power $P_{r 4}$ in our further calculation which will be discussed in experiment section.

\section{Power Distribution}

In spite of the residual power effect, another factor that might degrade the signal performance after WE II is the exchanged signal power. Ideally, without considering any other loss or gain effect except SPM, XPM, and FWM effect among WE II, the exchanged signal power will equal to original power if the total power $P_{0}$ is set at values such as point $\mathrm{B}$ in Fig. 4 . If we fix the pump power and increase the signal power like what we do in investigating the dynamic range in following sections, the total power almost equals to the pump power and will only slightly vary when the signal power is low. However, when the signal power is high, if the signal powers keep increasing, the total power will deviate from point $\mathrm{B}$, entering into the dotted box C. If so, the power of one exchanged signal may be smaller than that of its initial power, resulting in its Q-factor reduction, such as the exchanged $S 1\left(P_{4}\right)$ in Fig. 4. In Fig. 4, we calculated the normalized signal output powers $P_{4}(L) / P_{4}(0)$ and $P_{3}(L) / P_{3}(0)$ as a function of the input total power. The fiber parameters were the same as those used to obtain Fig. 2 except that $L=1 \mathrm{~km}$ and pump power was a variable. To overcome the undesired power distribution, in our experiment, we tuned the pump power in order to enhance the wavelength exchange.

\section{E. Dynamic Range}

We have discussed the importance of investigating the dynamic range of WE II in the introduction section. In this subsection, we will analyze it theoretically. Our studies are classified into two cases. First, we assume equal powers of the two signals. By adjusting the input power of the signals, the dynamic performance of WE II can be investigated [Fig. 5(a)]. Second, we study the performance of WE II when operating under imbalanced signal powers which reassembles practical networking systems [Fig. 5(b)]. In this case, one of the signals' powers are (a)

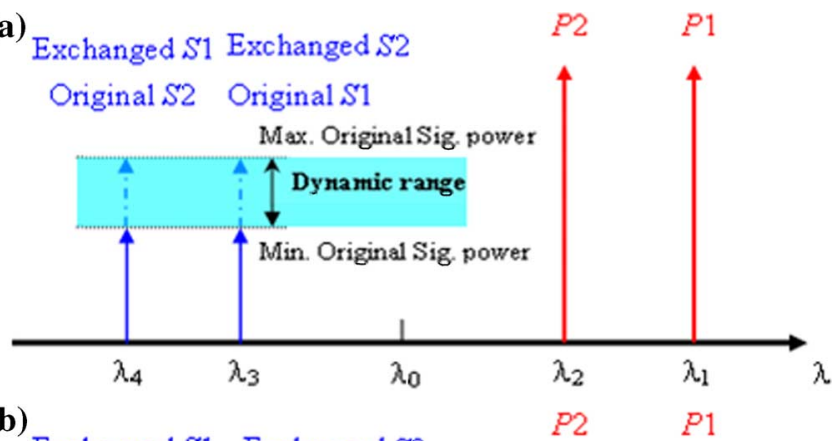

(b)

Exchanged S1 Exchanged S2

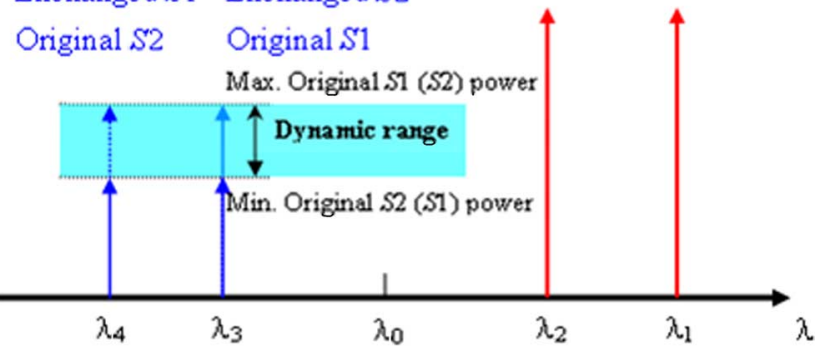

Fig. 5. Proposed approach for investigating the dynamic performance of the WE II with (a) balanced signal powers and (b) imbalanced signal powers.

higher than that of the others and the dynamic range is considered as the tolerable power difference between the two input signals which can maintain a good performance of WE II.

With the solution for $\mathrm{WE} \mathrm{II}_{\perp}$ and its noise characteristics discussed above, we can predict the dynamic range performance of WE II, which is plotted in Fig. 6. Fig. 6(a) plots the case of balanced signal powers, while Fig. 6(b) plots the case of imbalanced signal powers. The fiber and pump parameters used were the same as those used to obtain Fig. 2 except that the signal powers were variables and fiber length was $1 \mathrm{~km}$. The input extinction ratio $r_{\text {ex,in }}$ and the output extinction ratio $r_{\text {ex,out }}$ were obtained from experiments so that the calculated Q-factor was more practical. The signal input power stands for the initial total signal power $P_{3}(0)+P_{4}(0)$ in Fig. 6(a). The magnitude of power difference at the input was defined as $\Delta P=P_{3}(0)-$ $P_{4}(0)$ while the total signal power was maintained at $6 \mathrm{dBm}$ in Fig. 6(b). In order to investigate the exchange performance, the Q-factors of the exchanged signals were compared with the original ones. The Q-factor difference between the original signal and exchanged one is called Q-factor penalty as in (15).

As shown in Fig. 6(a), when the signal power is low, the Q-factor penalty of $S 1$ is slightly lower than that of $S 2$. With the signal power increasing, the Q-factor penalty of $S 1$ is also increasing but that of $S 2$ are decreasing until the signal power approaches $9 \mathrm{dBm}$. At high signal power region, the penalty of $\mathrm{S} 1$ is higher than that of $\mathrm{S} 2$. The divergence between two signal penalties is believed to be caused by residual power at respective wavelength. Table I lists the variation of penalties of signals in both conditions and that of their sources. In the case of balanced signal power, the magnitude of $\kappa_{1}$ first decreases, then increases because the positive signal power in the brackets of (27) compensates for the negative $-\Delta \beta$ ( $\Delta \beta>0$ is assumed), which is shown in the entry $\left|\kappa_{1}\right|$ in Table I. On the other hand, $\kappa_{2}$ is increasing within the whole dynamic range for the nonlinear term can never compensate for the positive $\Delta \beta$ as in (28). At low signal power region, the residue difference at two wavelengths is 

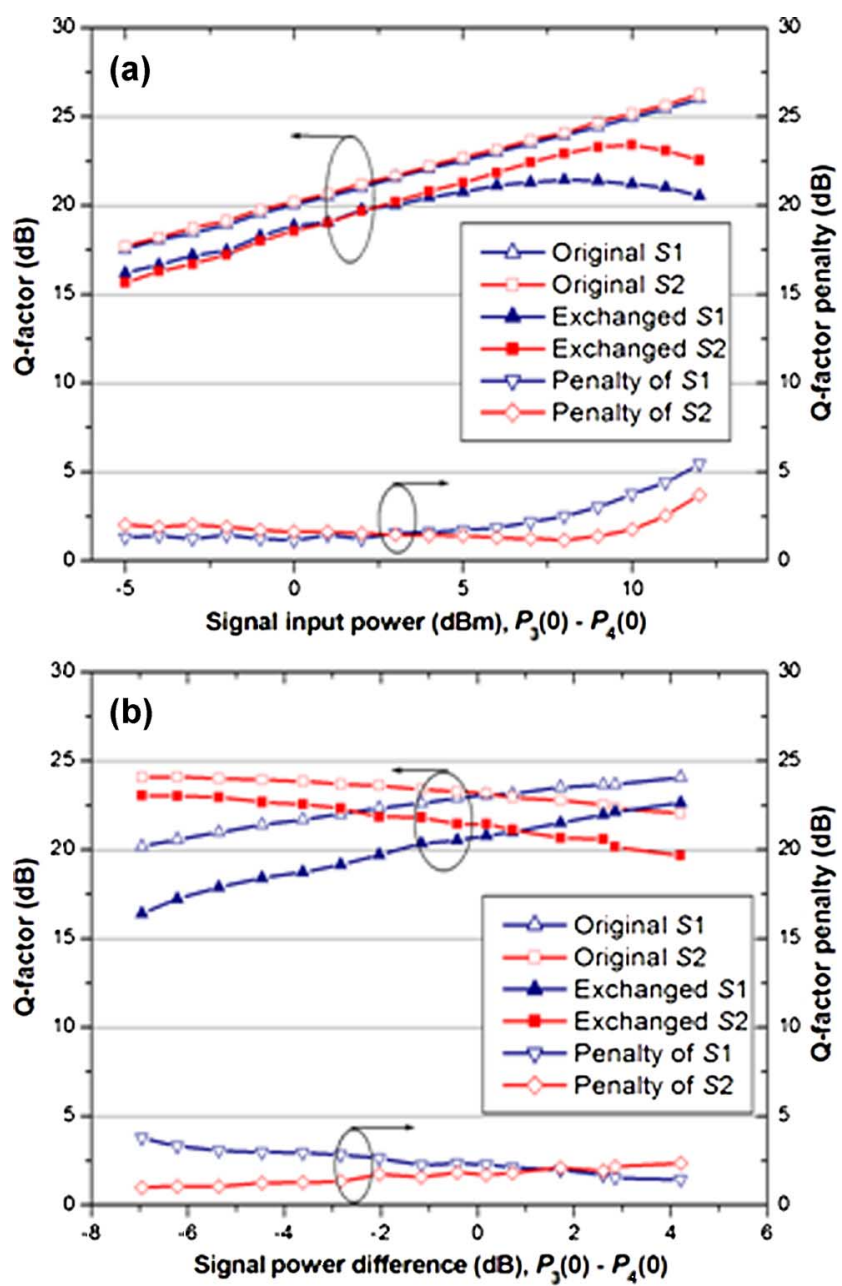

Fig. 6. Calculated Q-factor penalties among exchanged signals with (a) balanced and (b) imbalanced input powers.

TABLE I

THe VARiation of Penalties AND Their Sources

\begin{tabular}{|c|c|c|c|c|c|c|}
\hline \multicolumn{2}{|c|}{$\begin{array}{c}\text { Signals in various } \\
\text { cases }\end{array}$} & $\begin{array}{c}\text { Input } \\
\text { power }\end{array}$ & $\left|\kappa_{i}\right|$ & Residue & $\begin{array}{c}\text { Output } \\
\text { power }\end{array}$ & Penalty \\
\hline $\begin{array}{c}\text { Balanced } \\
\text { signal }\end{array}$ & $S 1$ & $P_{3}(0) \uparrow$ & $\left|\kappa_{2}\right| \uparrow$ & $P_{r 4} \uparrow$ & $P_{4}(L) \uparrow$ & $\uparrow$ \\
\cline { 2 - 7 } & $S 2$ & $P_{4}(0) \uparrow$ & $\left|\kappa_{1}\right| \downarrow \uparrow$ & $P_{r 3} \downarrow \uparrow$ & $P_{3}(L) \uparrow$ & $\downarrow \uparrow$ \\
\hline $\begin{array}{c}\text { Imbalanced } \\
\text { signal }\end{array}$ & $S 1$ & $P_{3}(0) \uparrow$ & $\left|\kappa_{2}\right| \rightarrow$ & $P_{r 4} \downarrow$ & $P_{4}(L) \uparrow$ & $\downarrow$ \\
\cline { 2 - 7 } & $S 2$ & $P_{4}(0) \downarrow$ & $\left|\kappa_{1}\right| \rightarrow$ & $P_{r 3} \rightarrow$ & $P_{3}(L) \downarrow$ & $\uparrow$ \\
\hline
\end{tabular}

not obvious due to the fact that the magnitudes of $\kappa_{1}$ and $\kappa_{2}$ are almost the same (i.e., $\kappa_{1}=-\kappa_{2}$ or $P_{r 3} \approx P_{r 4}$ ). Consequently, the penalty of $S 1$ nearly equals to that of $S 2$ until the total signal input power is around $3 \mathrm{dBm}$. When the signal power is high, especially when the phase-mismatch parameter $\kappa_{1}$ almost equals to zero (at around $6 \mathrm{dBm}$ ), $P_{r 3}$ is quite low, which contributes to the lower penalty of $S 2$. On the contrary, the residue $P_{r 4}$ rises over the whole dynamic range due to the increasing $\kappa_{2}$. This explains the rising trend behavior of the penalty of $S 1$.

In Fig. 6(b), we noticed that when $\Delta P$ increases, the penalty of $S 1$ is decreasing but that of $S 2$ is slightly increasing. These two penalty curves are almost converged from -2 to $3 \mathrm{dBm}$. Similar analysis centered on residual power can be applied to clarify the exchange performance in the imbalanced signal case.
One difference from the balanced signal case is that $\kappa_{1}$ and $\kappa_{2}$ are constant because the total signal power $P_{3}(0)+P_{4}(0)$ is fixed, so the variation of residual power $P_{\mathrm{r} 3}$ and $P_{\mathrm{r} 4}$ is dominated by input signal power $P_{3}(0)$ and $P_{4}(0)$ as the three discrete signal power we compared in Fig. 3. Consequently, $S 1$ 's penalty decreases; whereas, the penalty of $S 2$ does not vary much because the choice of signal power (around $6 \mathrm{dBm}$ ) makes $\kappa_{1}$ very small over the dynamic range.

Despite of the above detailed investigation about the exchange performance at both signal wavelengths, these theoretical predictions demonstrate that the performance of WE II does not degrade greatly over the dynamic range we investigated. These results, which strengthen our confidence on the practical usage of wavelength exchange, will be verified experimentally in the following section.

\section{EXPERIMENT}

\section{A. Experimental Setup}

We now investigate the dynamic range of WE II by experiments and compare it with the theoretical value in the previous section. The experimental setup is shown in Fig. 7. The wavelength exchange consisted of a $1 \mathrm{~km}$ of HNL-DSF with a $\lambda_{0}$ of $1541 \mathrm{~nm}$, a dispersion slope of $0.03 \mathrm{ps} / \mathrm{nm}^{2} \mathrm{~km}$ and a fiber nonlinearity coefficient $\gamma$ of $12 \mathrm{~W}^{-1} \mathrm{~km}^{-1}$. The two pumps were prepared by two tunable laser sources, TLS 1 and TLS 2, which were fixed at 1549 and $1554 \mathrm{~nm}$, respectively. They were phase-modulated (PM) by $10 \mathrm{~Gb} / \mathrm{s} 2^{23}-1$ pseudorandom bit sequence (PRBS) to suppress stimulated Brillouin scattering (SBS) [15]. EDFA 1 served as the preamplifier to a booster EDFA 2. Two tuneable bandpass filters (TBPF) with 2-nm bandwidth were inserted after EDFA 1 so as to filter out the two pumps separately and reduce ASE noise. Two polarization controllers (PC 3 and 4) were used to control the SOP of the two pumps such that orthogonal pump configuration could be maintained by minimizing the power of the spurious FWM components. The two pumps were then combined with the use of a WDM coupler. Wavelengths of the two signals, prepared by TLS 3 and TLS 4, were fixed at 1534 and $1529 \mathrm{~nm}$, respectively. They were amplitude-modulated with a $10 \mathrm{~Gb} / \mathrm{s} 2^{23}-$ 1 PRBS. PC 6 and 8 aligned the signals with the orthogonal pumps. EDFA 3, with a gain bandwidth of 1528-1560 nm, was used to compensate for the insertion losses of the amplitude modulators (AMs) and 50/50 coupler. VOA 1 was inserted after EDFA 2 to adjust the input pump powers; while VOA 2 was used to control the input signal powers launching into the HNL-DSF to investigate the dynamic range performance of $\mathrm{WE} \mathrm{II}_{\perp}$.

An $80 / 20$ coupler combined $80 \%$ of the pump powers and $20 \%$ of the signal powers. In our experiment, the input powers of the two pumps were measured to be 20 and $20.4 \mathrm{dBm}$, while the powers of the two signals were varied between -5 and $12 \mathrm{dBm}$. The reason why the pump power in experiment is slightly lower than that of theoretical value is probably due to the reason that the initial neighboring waves were not strictly orthogonal to each other or the birefringence within the fiber, which slightly enhance the FWM efficiency. A TBPF was used at the fiber output to filter out the exchanged signals. They were then sent 


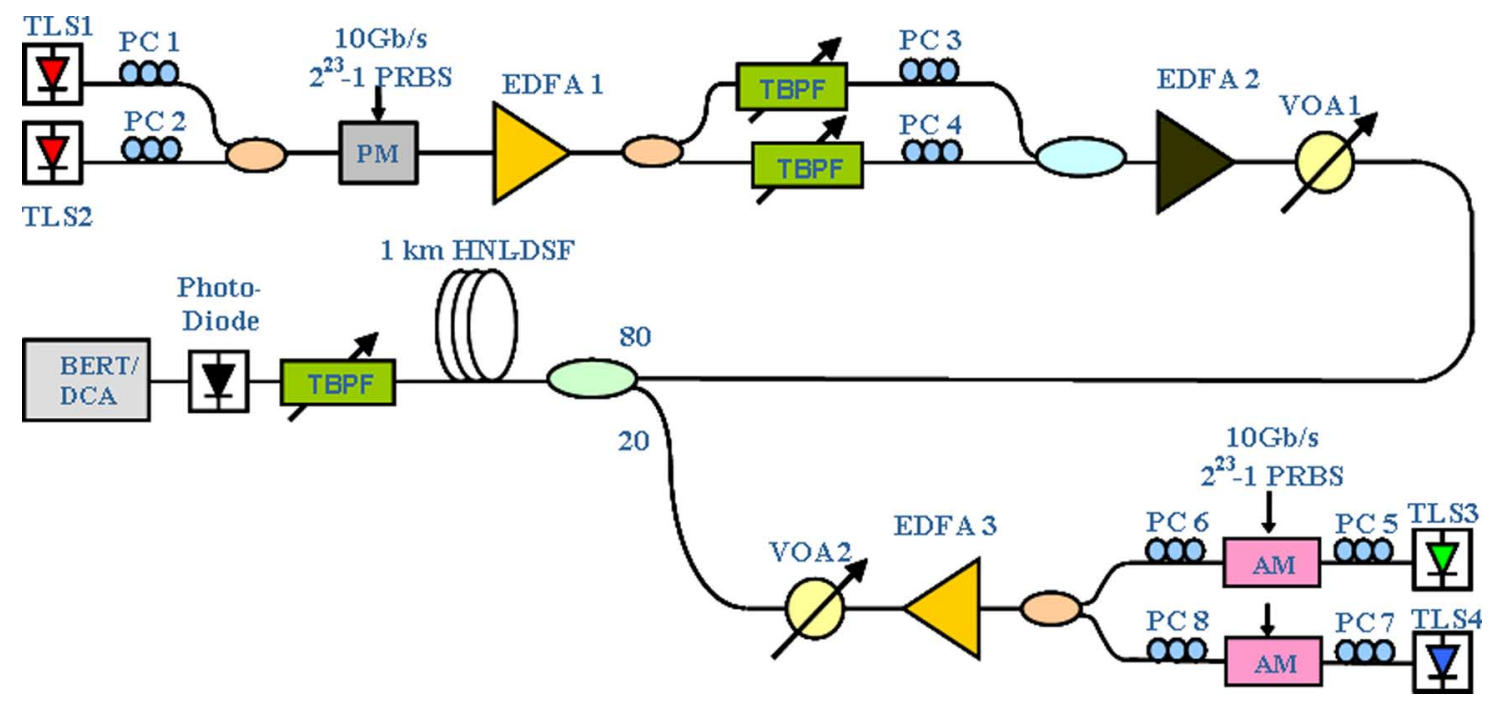

Fig. 7. Experimental setup of the wavelength exchange.

to the digital communication analyzer (DCA) and BER tester (BERT) for eye diagrams and BER measurements, respectively.

\section{B. Balanced Signal Powers}

The Q-factors and extinction ratios (ERs) of the exchanged signals over the dynamic range are plotted in Fig. 8. The signal input power at the $x$-axis stands for the total input signal power $P_{3}(0)+P_{4}(0)$. It shows that good performance of WE can be successfully maintained over a wide dynamic range of $17 \mathrm{~dB}$ ( -5 to $12 \mathrm{dBm}$ ) with Q-factors $>15 \mathrm{~dB}$ and ERs of the eyes $>10 \mathrm{~dB}$. The dynamic range was limited by the output power of EDFA 3 and the sensitivity of the photodetector in our experiment, otherwise a wider dynamic range of WE could be recorded. It can be observed that the Q-factor curves qualitatively match with our calculated curves as shown in Fig. 6(a). It verifies our analysis about the mechanisms through which residual signal interfere with the exchanged one. In the experiment, the performance of WE was also degraded at relative high or low signal levels due to stronger spurious FWM terms and poor signal-to-noise ratio (SNR), respectively, that had not been accounted in the analysis.

The receiver sensitivities of the exchanged signals were measured and compared with their corresponding original signals. We considered three cases, they were signal powers at 1) $-5 \mathrm{dBm}$, 2) $6 \mathrm{dBm}$, and 3) $12 \mathrm{dBm}$, which corresponded to the best (case 2) and the worst cases (cases 1 and 3) at the edges of the available dynamic range. The calculated residual power $P_{r 4}$ from original $S 2$ at these three power cases are already shown in Fig. 3. Their eye diagrams are shown in Fig. 9. They illustrate that clear eye openings are observed in all cases. The measured BER curves for the exchanged signals are plotted in Fig. 10. At BER of $10^{-9}$, the receiver sensitivities of the original signals at 1534 and $1529 \mathrm{~nm}$ are -27.2 and $-27.8 \mathrm{dBm}$, respectively, while that of the exchanged signal were measured to be -26.5 and $-27 \mathrm{dBm}$, respectively, in the best case (i.e. case 2). The power penalties incurred in the wavelength exchange are $<1 \mathrm{~dB}$. In cases 1 and 3 , the power penalties slightly increased to $\sim 2 \mathrm{~dB}$.
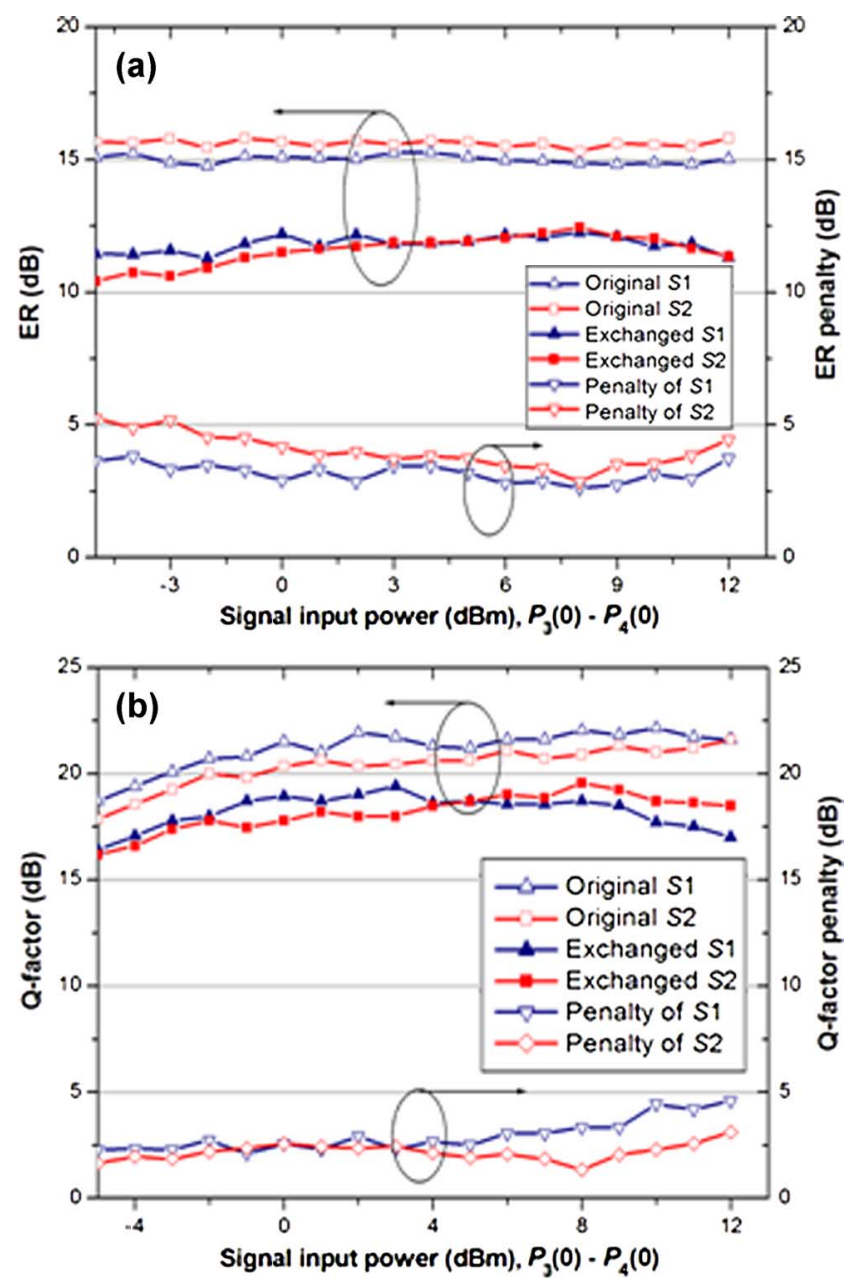

Fig. 8. (a) ERs and (b) Q-factors among exchanged signals with balanced input powers.

\section{Imbalanced Signal Powers}

We then investigated the dynamic range under imbalanced signal powers condition. The power differences between the two input signals at 1534 (original $S 1$ ) and $1529 \mathrm{~nm}$ (original $S 2$ ) 


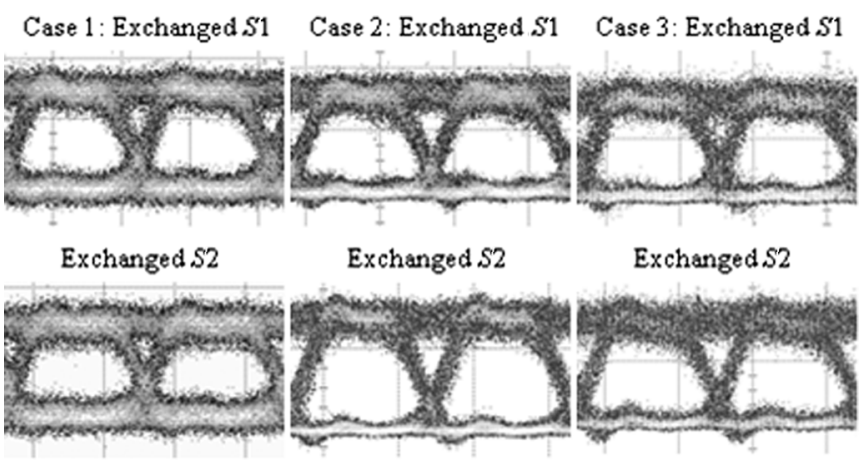

Fig. 9. Measured eye diagrams of the exchanged signals in the three cases.

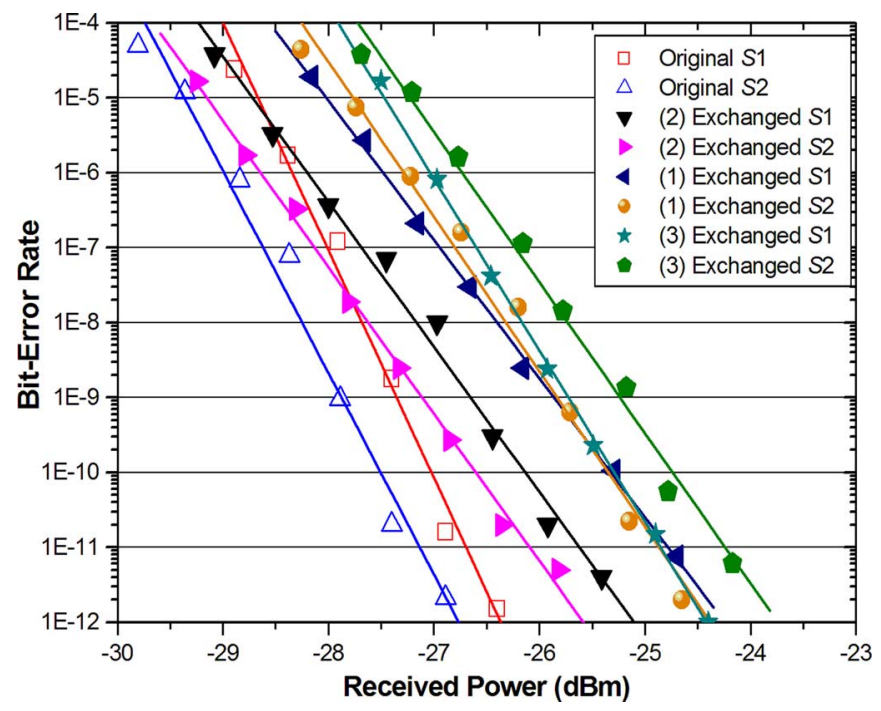

Fig. 10. Measured BER curves for the exchanged signals before and after WE in the three cases.

were defined as $\Delta P=P_{3}(0)-P_{4}(0)$ which were measured at the fiber input. It was done by varying the signal power output from TLS 3, keeping the output power of TLS 4 constant; while maintained the total signal power after EDFA at $6 \mathrm{dBm}$. The ERs and Q-factors of the exchanged signals over the tuning range are plotted in Fig. 11. The experimental Q-factor penalties can be maintained below $5 \mathrm{~dB}$ over a dynamic range of $8 \mathrm{~dB}(-5$ to $3 \mathrm{~dB}$ ), which showed a good agreement with the calculated penalty as shown in Fig. 6(b).

The receiver sensitivities of the two exchanged signals at $\Delta P$ of $-5 \mathrm{~dB}$ are measured. Error-free WE could not be recorded beyond this value $(<-5 \mathrm{~dB})$ due to poor SNR of EDFA 3 and severe crosstalk between the exchanged signals resided at the wavelength possessing high input signal power. The eye diagrams of exchanged signals in this condition are shown in Fig. 12. The measured BER curves are plotted in Fig. 13. At BER of $10^{-9}$, the receiver sensitivity of the exchanged signal at $1529 \mathrm{~nm}$ was around $-22 \mathrm{dBm}$. The power penalty incurred is $\sim 2 \mathrm{~dB}$; while negligible power penalty was recoded at the exchanged signal at $1534 \mathrm{~nm}$ owing to low crosstalk between the strong signal and weak residue. Similar performance was observed at $\Delta P$ of $3 \mathrm{~dB}$.
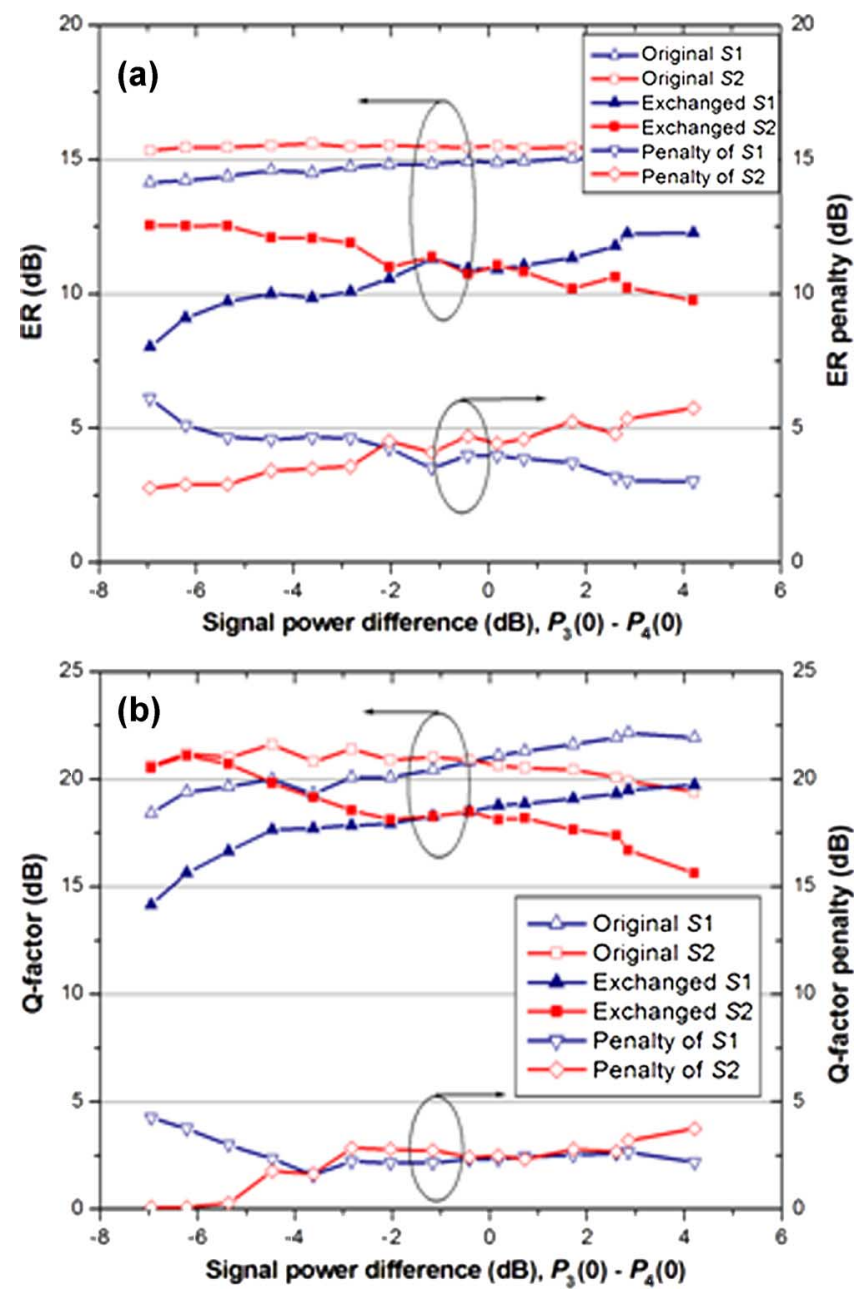

Fig. 11. (a) ERs and (b) Q-factors among exchanged signals with imbalanced input powers.
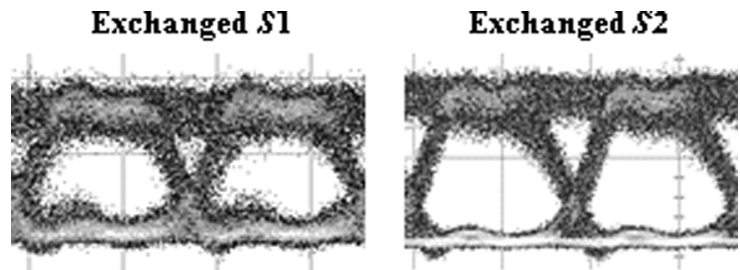

Fig. 12. Measured eye diagrams of the exchanged signals after WE with a power difference of $-5 \mathrm{~dB}$.

\section{CONCLUSION}

In conclusion, the residual powers at the original wavelength constitute a major limiting factor for the exchanged signal quality in WE II. We have investigated its influence on the performance of WE II both analytically and experimentally over a wide dynamic range for both balanced and imbalanced signal power. Experimental results show that when the WE II was operated under balanced signal powers, the Q-factor penalty could be maintained below $5 \mathrm{~dB}$ over a dynamic range around $17 \mathrm{~dB}$. BERs of $<10^{-9}$ were maintained with power penalties of at most $2 \mathrm{~dB}$. In the case of imbalanced signal powers, the Q-factor penalty could be maintained below $5 \mathrm{~dB}$ 


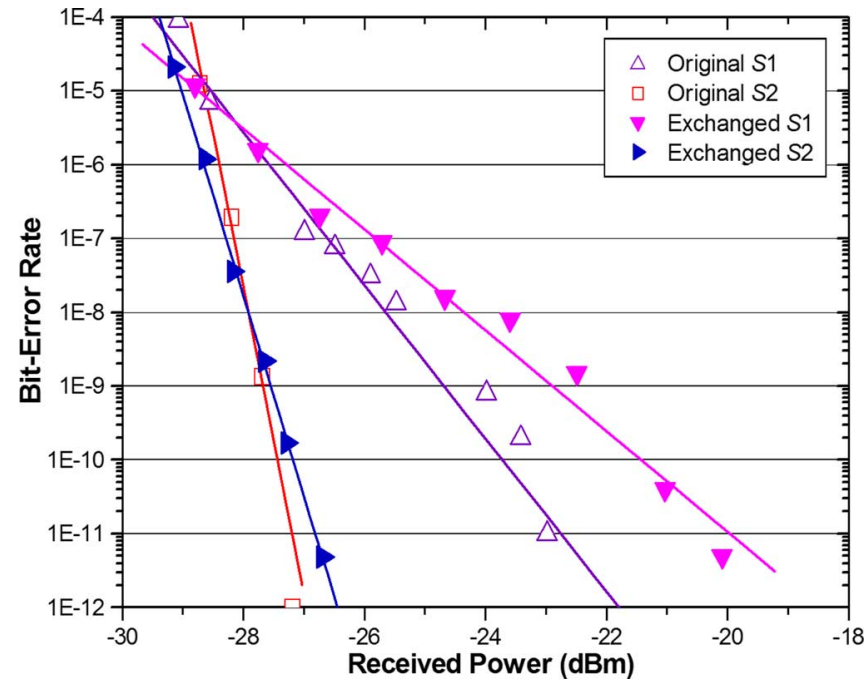

Fig. 13. Measured BER curves for the exchanged signals before and after WE with a power difference of $-5 \mathrm{~dB}$.

over a dynamic range wider than $8 \mathrm{~dB}$. The results verified our analysis about the mechanisms through which the residual signals mix with exchanged one. It provides a more comprehensive insight into the performance of WE II when operated under practical conditions. The theory shed light on the origin of residual power and the condition for complete exchange when considering only SPM, XPM, and FWM effect. The incomplete signal exchange problem can be solved to a large extent by satisfying phase mismatch condition which is analogous with that of FOPA. This theoretical model does not cover any ZDW fluctuation. It will render $\Delta \beta$ random and transform (3) and (4) into two stochastic differential equations with multiplicative noise whose solution generally requires a numerical approach [16]-[18]. This will be the subject of further research.

\section{ACKNOWLEDGMENT}

The authors would like to acknowledge Sumitomo Electric Industries for providing the HNL-DSF.

\section{REFERENCES}

[1] M. E. Marhic, Y. Parker, F. S. Yang, and L. G. Kazovsky, "Widely tunable spectrum translation and wavelength exchange by four-wave mixing in optical fibers," Opt. Lett., vol. 21, no. 23, pp. 1906-1908, 1996.

[2] K. Uesaka, K. K. Y. Wong, M. E. Marhic, and L. G. Kazovsky, "Wavelength exchange in a highly nonlinear dispersion-shifted fiber: Theory and experiments," IEEE J. Sel. Topics Quantum Electron., vol. 8, no. 3, pp. 560-568, May/Jun. 2002.

[3] K. K. Y. Wong, M. E. Marhic, K. Uesaka, and L. G. Kazovsky, "Wavelength exchange: A novel function for optical networks," Inf. Sci., no. 149, pp. 161-169, 2003

[4] R. W. L. Fung, H. K. Y. Cheung, and K. K. Y. Wong, "Widely tunable wavelength exchange in anomalous-dispersion regime," IEEE Photon. Technol. Lett., vol. 19, no. 22, pp. 1846-1848, Nov.15 2007.
[5] H. K. Y. Cheung, R. W. L. Fung, C. H. Kwok, and K. K. Y. Wong, "All-optical packet switching by pulsed-pump wavelength exchange in a highly nonlinear dispersion-shifted fiber," presented at the Proc. OFC, 2007, Paper OTuB4.

[6] O. F. Yilmaz, L. Christen, X. Wu, S. R. Nuccio, I. Fazal, and A. E. Willner, "Time-slot interchange of $40 \mathrm{Gbits} / \mathrm{s}$ variable length optical packets using conversion-dispersion-based tunable delays," Opt. Lett., vol. 33, no. 17, pp. 1954-1956, 2008.

[7] S. Radic, C. J. Mcknstrie, R. M. Jopson, A. H. Gnauck, J. C. Centanni, and A. R. Chraplyvy, "Multiple-band bit-level switching in two-pump fiber parametric devices," IEEE Photon. Technol. Lett., vol. 16, no. 3, pp. 852-854, Mar. 2004.

[8] Q. Lin, R. Jiang, C. F. Marki, C. J. Mckinstrie, R. Jopson, J. Ford, G. P. Agrawal, and S. Radic, " $40-\mathrm{Gb} / \mathrm{s}$ optical switching and wavelength multicasting in a two-pump parametric device," IEEE Photon. Technol. Lett., vol. 17, no. 11, pp. 2376-2378, Nov. 2005.

[9] C. H. Kwok, P. P. Kuo, and K. K. Y. Wong, "Pulsed pump wavelength exchange for high speed signal de-multiplexing," Opt. Express., vol. 16, no. 15, pp. 10894-10899, 2008.

[10] M. E. Marhic, Fiber Optical Parametric Amplifiers, Oscillators and Related Devices. Cambridge, U.K.: Cambridge Univ. Press, 2008, ch. 3.

[11] G. P. Agrawal, Fiber-Optic Communication Systems, 3rd ed. San Diego, CA: Academic, 2001, ch. 4.

[12] Y. J. Chen, "Four-wave mixing in optical fibers: Exact solution," J. Opt. Soc. Amer. B, vol. 6, no. 11, pp. 1986-1993, 1989.

[13] G. Cappellini and S. Trillo, "Third-order three-wave mixing in singlemode fibers: Exact solutions and spatial instability effects," J. Opt. Soc. Amer. B, vol. 8, no. 4, pp. 824-838, 1991.

[14] J. Hansryd, P. A. Andrekson, M. Westlund, J. Li, and P. O. Hedekvis, "Fiber-based optical parametric amplifiers and their applicaitons," IEEE J. Sel. Topics Quantum Electron., vol. 8, no. 3, pp. 506-519, Mar. 2002.

[15] S. K. Korotky, P. B. Hansen, L. Eskildsen, and J. J. Veselka, "Efficient phase modulation scheme for suppression of stimulated Brillouin scattering," presented at the IOOC 1995, 1995, paper WD2-1, 109-111.

[16] F. Yaman, Q. Lin, S. Radic, and G. P. Agrawal, "Impact of dispersion fluctuations on dual-pump fiber-optic parametric amplifiers," IEEE Photon. Technol. Lett., vol. 16, no. 5, pp. 1292-1294, May 2004.

[17] M. Karlsson, "Four-wave mixing in fibers with randomly varying zero-dispersion wavelength," J. Opt. Soc. Amer. B, vol. 15, no. 8, pp. 2269-2275, 1998.

[18] C. W. Gardiner, Handbook of Stochastic Methods, 2nd ed. New York: Springer-Verlag, 1985, ch. 4.

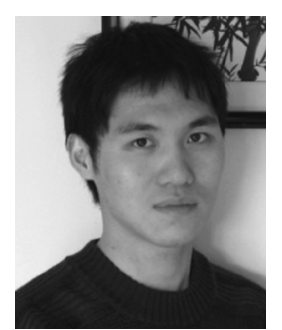

Mengzhe Shen received the B.S. degree in physics from the Shanghai Jiaotong University, Shanghai, China, in 2007. He is currently working towards the M.Phil. degree in electronic engineering at the University of Hong Kong.

His current research interests include applications of fiber-optical parametric effect and fiber nonlinearity in optical communication systems.

Henry King Yin Cheung, photograph and biography not available at the time of publication.

Rebecca Wai Lam Fung, photograph and biography not available at the time of publication. 


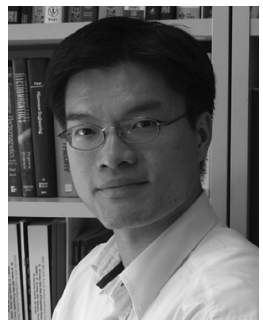

Kenneth Kin-Yip Wong (M'03) received combined B.E. (first-class honors with medal award) degree in electrical engineering and B.S. degree in physics from the University of Queensland, Brisbane, Australia, in 1997 and the M.S. and Ph.D. degrees in electrical engineering, from Stanford University, Stanford, CA, in 1998 and 2003, respectively.

$\mathrm{He}$ is currently an Assistant Professor in the Department of Electrical and Electronic Engineering, University of Hong Kong. He was a member of the Photonics and Networking Research Laboratory, Stanford University. His research field included DWDM systems, SCM optical systems, fiber nonlinearity, fiber-optical parametric amplifiers, and photonic crystal fibers. He is author or coauthor of over 100 journal and conference papers. He was with Hewlett-Packard Laboratories as Research Engineer and contributed in projects included parallel optics and VCSEL from 1998-1999. $\mathrm{He}$ also was an independent consultant with Innovation CORE (a Sumitomo Electric Company), CA, in 2004. He is a reviewer for Optics Letters, JOSA B, and Optics Express, IEE Electronics Letters, and Optics Communications.

Dr. Wong was the recipient of OSA New Focus Student Award and IEEE/ LEOS Graduate Student Fellowship, both in 2003. He is a reviewer for the IEEE Photonics TeChNOLOGY LETTERS and IEEE/OSA JouRNAL OF LIGHTWAVE TECHNOLOGY. He won the Best Teacher Award in 2005 and 2006 while with the Department of Electrical and Electronic Engineering, University of Hong Kong. $\mathrm{He}$ is a member of the OSA and the IEEE Lasers and Electro-Optic Society (LEOS). 